\title{
Metabolic Profiling of Borrelia in Ticks and in Whole Blood Samples by Headspace Gas Chromatography - A Case Report
}

\author{
Bruno Kolb ${ }^{1 *}$, E Marion Schneider ${ }^{2}$, Lorina Riesterer ${ }^{1}$, Leona Bier ${ }^{1}$ and Tabea Hein ${ }^{2}$ \\ ${ }^{1}$ Student Research Centre, Überlingen, Obertorstrasse, Germany \\ ${ }^{2}$ Division of Experimental Anesthesiology, University Hospital Ulm, Germany
}

*Corresponding author: Bruno Kolb, Student Research Centre, Obertorstrasse, Überlingen, Gremany, Tel: 49755163729 , Email: kolbbn@t-online.de

To Cite This Article: Bruno Kolb, Metabolic Profiling of Borrelia in Ticks and in Whole Blood Samples by Headspace Gas Chromatography - A Case Report. Am J Biomed Sci \& Res. 2019 - 6(4). AJBSR.MS.ID.001057. DOI: 10.34297/AJBSR.2019.06.001057.

Received: 監 November 25, 2019; Published: 監 December 05, 2019

Abstract

The technique of static headspace gas chromatography (HS-GC) was applied to monitor the emission of hydrogen and carbon dioxide from bacterial cultures to detect bacterial contamination in ticks and the transfer to humans, causing Lyme borreliosis. While the standard serological methods to recognize Lyme-disease rely upon the indirect determination of antibodies, the present method enables the direct proof of bacteria both in ticks and in the blood of infected patients, due to the specific hydrogen emission. Moreover, the progress of an applied therapy can be monitored while antibodies, once created, remain in the blood regardless of whether the applied therapy was successfully finished or not. This method was also used to compare the efficacy of chemical and natural antibiotics. The most efficient natural antibiotics found were oil of cloves and garlic extract preparations. It was found that the duration of a successful therapy with chemical and natural antibiotics varied but can be perfectly monitored by the gas chromatographic technique.

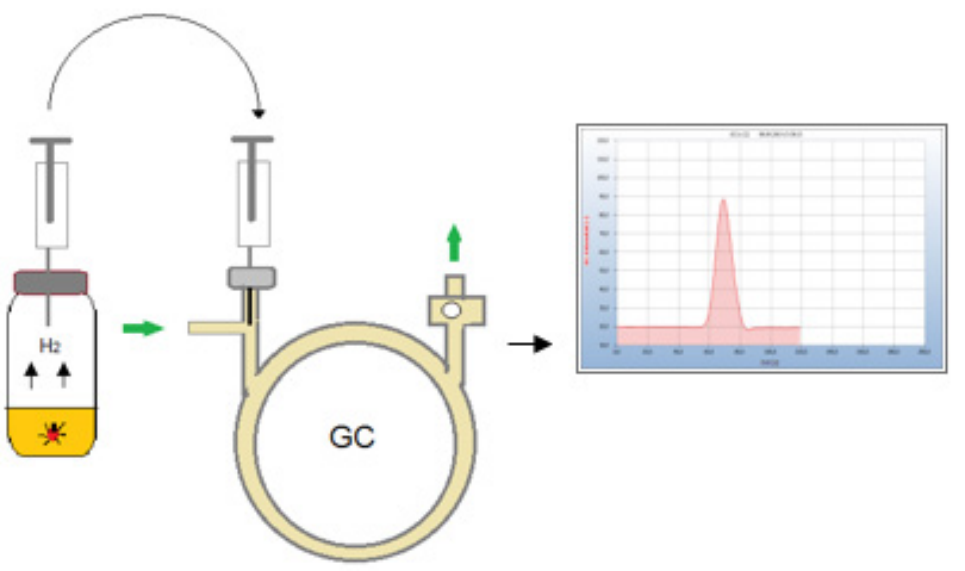

Graphical Abstract

Ticks and blood samples are inserted into the nutrient medium in a headspace vial and incubated for three days at $35^{\circ} \mathrm{C}$. An aliquot of the headspace is transferred to the gas chromatograph by a gas tight syringe. A hydrogen peak in the resulting chromatogram indicates contamination by Borrelia bacteria.

Keywords: Borrelia; Lyme disease; Headspace gas chromatography; Antibiotics; Antibiotic resistance, garlic.

List of Abbreviations: EM (Erythema migrans); GC (gas chromatography); HS-GC (headspace gas chromatography); Polytetrafluoroethylene (PTFE); volatile organic compounds (VOCs) 


\section{Introduction}

Bacteria emit volatile compounds, which are amenable to analysis by static headspace gas chromatography (HS-GC) if cultured in nutrient medium in the septum vials used for HS-GC. The technique of HS-GC has been applied since 1965 in examining the growth of bacteria in milk [1]. The class of compounds that receiving the most study was volatile organic compounds (VOCs), and the unique volatile mixture emitted may be used to identify infectious microbes to the species, and possibly the strain level [24]. Moreover, the impact of antibiotics on the emission of VOCs, if added to the microbial cultures, enables to test their antibacterial activity. N.J. Hayward investigated bacterial resistance of Clostridia from urinary tract infections against antibiotics by monitoring ethanol formation [5]. Antimicrobial resistance is a serious and current issue in medicine and novel antibiotics are needed to treat such multi-resistant microbes. HS-GC may be of interest to identify and characterize antimicrobial properties by chemical antibiotics as well as by phytochemicals and other natural compounds.

Such phytochemicals often contain several different classes of substances to combat multiple resistances of pathogens [6]. This approach requires many measurements and needs automation to improve efficacy. Gas chromatography is a well-established technique for automated qualitative and quantitative analysis of volatiles. In addition to the analysis of VOCs released from bacteria, both hydrogen $\left(\mathrm{H}_{2}\right)$ and carbon dioxide $\left(\mathrm{CO}_{2}\right)$ are highly informative metabolites. Obligate aerobes produce $\mathrm{CO}_{2}$ and water, while facultative anaerobes and microaerophilic anaerobes emit $\mathrm{H}_{2}$ and $\mathrm{CO}_{2}$ together with some other organic compounds, for example ethanol. Obligate anaerobes emit many VOCs and $\mathrm{H}_{2}$ and $\mathrm{CO}_{2}$ but need an oxygen-free atmosphere in the culture vial, because they would not survive atmospheric oxygen concentrations, but when air is replaced by nitrogen gas these bacteria can thrive.

By monitoring both, $\mathrm{H}_{2}$ and $\mathrm{CO}_{2}$ the class of bacteria can be identified [7]: peaks of $\mathrm{H}_{2}$ and $\mathrm{CO}_{2}$ in the chromatogram are produced by facultative anaerobes and by obligate anaerobes too, but the latter only in an oxygen-free atmosphere in the closed headspace vials, while obligate aerobes generate $\mathrm{CO}_{2}$ but no hydrogen peak. Our method is focused on hydrogen emitting facultative anaerobic and microaerophile bacteria only. We previously applied the analysis of $\mathrm{H}_{2}$ and $\mathrm{CO}_{2}$ emission profiles to study tick-borne disease related pathogens and tested the efficacy of antibiotics such as doxycycline and amoxicillin [8] against Lyme-disease. Tick-borne diseases are difficult to recognize since they often arise late after Borrelia infection and comprise a wide range of various Lyme disease symptoms, such as Lyme arthritis, myalgias, heart abnormalities, chronic fatigue, neuropsychiatric depression and several more. In some areas up to $25 \%$ of ticks contain Borrelia bacteria and from these only few percent are also supposed to cause infection. Borrelia are microaerophile and can thrive in an atmosphere with a reduced oxygen concentration compared to air. When the concentration of oxygen in the gas-tight culture vials declines by oxygen consumption they change from oxidative metabolism generating carbon dioxide and water, to fermentation with hydrogen emission. The closed headspace vials thus act just as a micro anaerobic incubator.

The objective of the current study compares the activity of chemical and natural antibiotics on contaminated ticks and on blood samples of infected patients. The HS-GC-analytic method can monitor both, disease progress as well as regression. Lyme disease is a potentially fatal illness transmitted through the bite of infected ticks. Most infected humans are cured by standard chemical antibiotics, such as doxycycline, if treated in due time following the bite by an infected tick [9]. However, recommendations for this antibiotic treatment depend on the documentation of a reactive skin lesion, namely Erythema migrans (EM). Lesions are the most common signs of infection, but in many cases these lesions do not occur, and a potential infection would not be recognized and therefore not be treated. About 10-20 \% of patients suffer from chronic pain caused by persistent borreliosis and tick-transmitted pathogens. All diagnostic devices available bear major drawbacks in terms of sensitivity and specificity [10]. Serological tests (Western Blot and ELISA) are invalid to determine the duration of antibiotic therapy necessary for treatment (two weeks up to several months). A simple and easy to handle technique is therefore highly desirable for monitoring the presence of bacteria in the blood of an infected patient and to control the efficacy of an antibiotic therapy. The method of HS-GC proposed in this study may meet this requirement in an excellent way. Lyme-disease, however, may not always be caused by Borrelia burgdorferi alone, but often by co-infection with subspecies of Borrelia (B. afzellii, B. garinii, B. spielmanii) or by other accompanying facultative bacteria such as Mycoplasma and Yersinia and some more [11]. Our GC-technique can only prove the presence of hydrogen-emitting facultative anaerobic bacteria and not a single species such as Borrelia burgdorferi and for this reason we prefer the term Borreliella as common genus, suggested by S.J. Cutler et al. [12]. This is reasonable because the proof of hydrogen comprises senso latu all those members of this species complex, which all together may be responsible for Lyme-disease.

\section{Materials and Methods}

\section{Materials and instrumentation}

Gas chromatograph GC-AK 11, Aug. Hedinger GmbH \& Co KG, Germany; GC-column: $0.8 \mathrm{~m}$ x $6 \mathrm{~mm}$ polyamide tube, Silica gel 60/80 mesh; headspace vials: $6 \mathrm{ml}$ from Perkin Elmer crimp-capped with PTFE-laminated butyl rubber septa. Nutrient medium: Merck Life Science GmbH, Germany, tryptic soy broth acc EP+ USP 3080r-20p; composition: pancreatic digest of casein, 17g; papaic digest of soya bean meal, $3 \mathrm{~g}$; sodium chloride, $5 \mathrm{~g}$; dipotassium hydrogen phosphate, 2.5g; glucose monohydrate. Sugar coated tablets with pulverized garlic Kwai@forte 300mg: Cassella-med GmbH \& Co.KG, Germany. 


\section{Interpretation of the chromatograms}

The gas chromatograph is operated with ambient air as carrier gas and using a heat conductivity detector. Depending on the differences in heat conductivity both positive and negative peaks are generated. Hydrogen, due to its high heat conductivity produces a positive peak with good sensitivity while carbon dioxide due to its lower heat conductivity a negative one. Two negative peaks are shown in the gas chromatograms, one close to the rear of the positive hydrogen peak and later a negative peak from carbon dioxide. Blood in the headspace vials is still active and converts the oxygen of the air in the vials to carbon dioxide, thus producing this negative peak at the end of the chromatogram. The small negative peak at the rear of the hydrogen peak is thus caused by a deficit of oxygen in the air in the vials, not only due to the oxygen consumption of blood in case of blood samples, but also to a smaller extent by some oxidation of the nutrient medium. The air sample withdrawn from the vial is thus enriched with nitrogen and produces this small negative peak at the rear of the hydrogen peak according to the retention time of nitrogen. Presentation of the chromatograms: $\mathrm{x}$-axis time in seconds [s]; y-axis gas phase concentration in arbitrary units.

\section{Method and sample preparation}

Volunteer donors with and w/o clinical signs of borreliosis provided blood samples by piercing a fingertip with a needle, except the example given in Figure 1-Bb, where the blood sample was withdrawn from the vain by a physician. The resulting droplet of blood $(\sim 50 \mu \mathrm{l})$ from the fingertip was sucked into a sterile cotton ball from a cotton bud in such a way that the cotton ball came not in contact with the skin and the blood-soaked cotton ball was then inserted into the sterile nutrient broth in the vial which was closed immediately by a crimp closure. Not infected blood samples produced no hydrogen peak because healthy blood contains no hydrogen emitting bacteria. Ticks were sampled from humans and house cats and are simply thrown into the nutrient broth. The charged vials are incubated in general for 3 days at $35^{\circ} \mathrm{C}$. Only if the GC analysis after 3 days incubation is negative is the time prolonged for another day, either to confirm the negative result or to allow more time for the slow reproduction of spirochetes in the case of a low concentration in blood to meet the GC detector sensitivity. The samples under investigation, either solid ticks or liquid samples and the cotton ball from a cotton bud as used for a smear, are cultured in $6 \mathrm{ml}$ septum vials containing $2.5 \mathrm{ml}$ sterilized nutrient medium. After the necessary time of incubation an aliquot of 0.3 $\mathrm{ml}$ is withdrawn from the gas phase by a gas tight syringe and the volatile compounds are analyzed by HS-GC. Antibiotics added to the bacterial cultures in the vials are effective if any gas emission is suppressed. If not, they are either ineffective or the related bacteria are resistant.

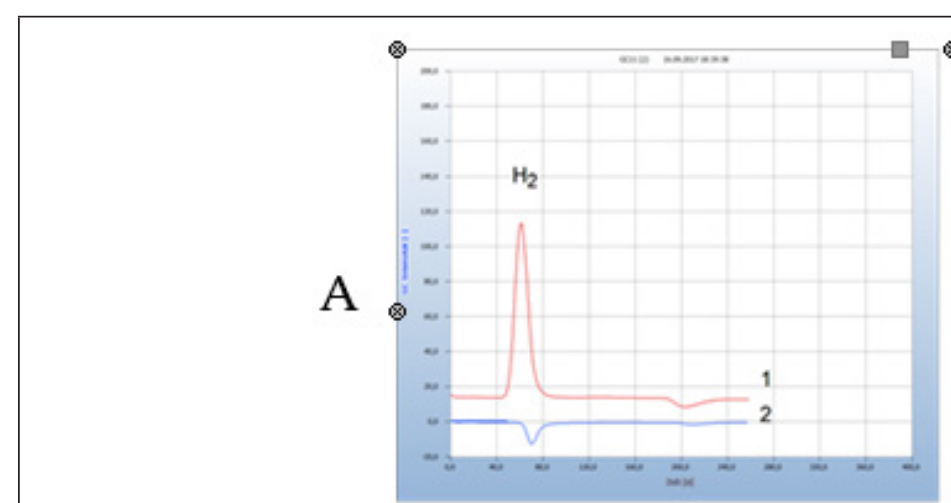

(a)

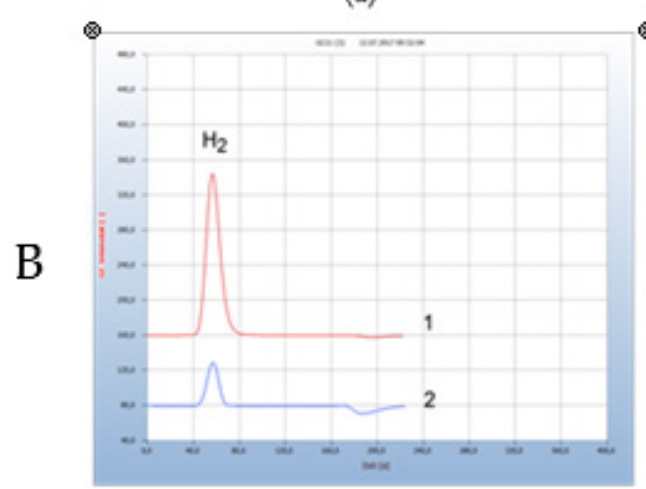

(a)

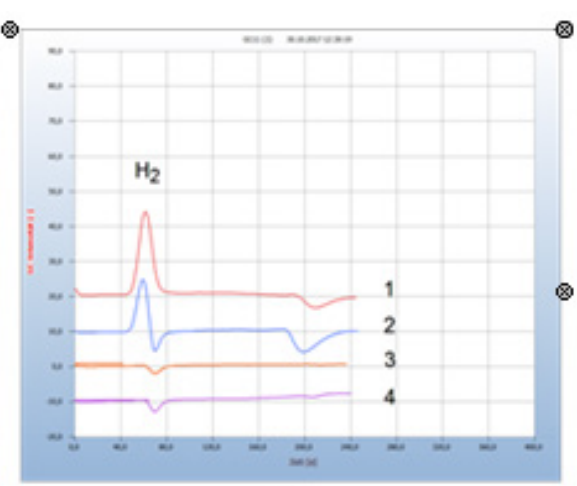

(b)

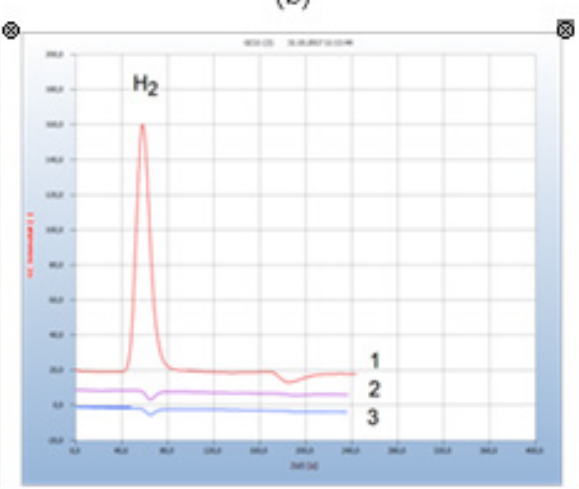

(b)

Figure 1: GC analysis of Borreliella in tick samples. A) (a) Borreliella in ticks. 1: contaminated; 2: non-contaminated. (b) Antibiotic resistance of contaminated ticks. 1: contaminated ticks, 2: contaminated ticks + Penicillin, 3: contaminated ticks + Doxycycline, 4: blank from sample-free bacterial broth. B) (a) Borreliella in blood and in the tick; 1 : $\mathrm{H}_{2}$ from infected blood sample, $2: \mathrm{H}_{2}$ from the responsible tick. (b) Borreliellain blood; 1: infected blood with Borreliella 2: normal healthy blood without Borreliella, 3: blank from cotton ball in nutrient medium. 


\section{Electron microscopy}

Bacterial cultures tested by HS-GC were fixed in $2.5 \%$ of glutardialdehyde (Sigma.com) followed by osmium tetroxide staining $(0.1 \%)$ and stepwise embedding of the samples in Epon (Fluka.com, USA) (polymerization at 333K within $72 \mathrm{~h}$ ). Ultrathin sections were cut using a microtome (Leica Ultracut CT ultramicrotome) with a diamond knife (Diatome, Switzerland). Specimen were counterstained with lead citrate and examined using a JEOL (JEOL.com, Germany) 1400 at $120 \mathrm{keV}$.

\section{Results}

\section{Borreliosis therapy with Doxycycline}

Ticks cultured in the nutrient medium emit hydrogen if they are contaminated by facultative or microaerophilic anaerobes.We have examined many ticks with the following result: about $20 \%$ emitted hydrogen and were apparently charged by such bacteria, while the rest (about 80\%) failed to generate hydrogen. This result agrees with the official statement that in our area about $20-30 \%$ are contaminated. The chromatograms from not contaminated ticks were in every case identical with the blank sample of a pure sterilized cotton ball in the nutrient broth. However, there might still be the possibility of contamination by other microbes such as aerobes or obligately anaerobes, but our method is specific for

hydrogen emitting facultative anaerobes such as Borreliella and ignores such microbes. Figure. 1-Aa presents such an example taken from many others: two ticks were inserted separately into two vials already containing the nutrient medium and were incubated for 3 days. One of the two ticks was contaminated as indicated by the emitted hydrogen while the other was found to be free of bacteria. Proof of bacterial load in ticks by HS-GC was already described [8]. The intention of this work was the extension of the GC-analysis technique for

i) evaluation of the effectiveness of antibiotic treatment and

ii) supporting the search for effective antimicrobial compounds.

Figure 1-Ab shows the comparison of two common antibiotics regarding their antimicrobial activity. Penicillin was found to be ineffective, while doxycycline, the standard antibiotic for Lyme borreliosis worked sufficiently well by complete disappearance of the characteristic hydrogen emission peak (Figure. 1-Ab). In further experiments, three ticks were crushed and incubated in $100 \mu \mathrm{l}$ of the sample-free nutrient medium, thereby assuming that at least one of them was contaminated. Aliquots of such a homogenous suspension were transferred into the nutrient medium in the vials containing the antibiotics to be examined.

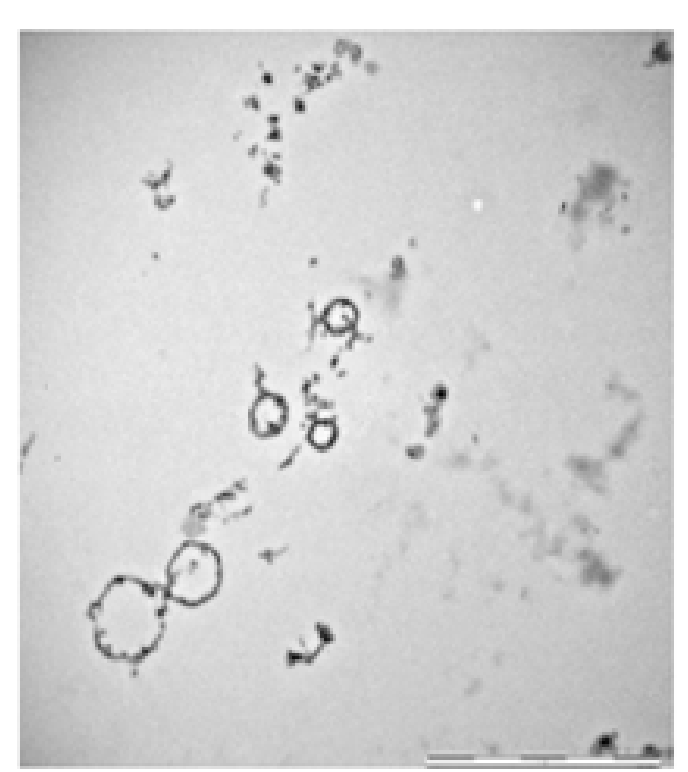

Figure 2: Trans electron microscopy of cultured Borrelia species. (a) Bacterial cultures tested by HS-GC were fixed in $2.5 \%$ of glutardialdehyde (Sigma.com) followed by osmium tetra-oxide staining $(0.1 \%)$ and stepwise embedding of the samples in Epon (Fluka.com, USA) (polymerization at $333 \mathrm{~K}$ within $72 \mathrm{~h}$ ). Ultrathin sections were cut using a microtome (Leica Ultracut UCT ultramicrotome) with a diamond knife (Diatome, Switzerland). Specimen were counterstained with lead citrate and examined using a JEOL (JEOL.com, Germany) 1400 at 120 keV.

Only few percent of contaminated ticks are known to infect the human target, due to inefficient transmission of the pathogen and only the analysis of a blood sample can unambiguously confirm a suspected borreliosis infection. Figure 1-Ba gives such an example of bacterial transfer to human blood after tick bite of a contaminated insect, shown by the hydrogen peak from the contaminated tick and in the blood sample. Blood normally contains no bacteria, and blood from healthy donors therefore was always found negative of hydrogen emitting bacteria. This is representatively shown in Figure $1-\mathrm{Bb}$ and compared with a contaminated blood sample from a patient who was bitten by a tick followed few weeks later by an erythema migrants which is the most common sign of an 
infection after a bite by a tick and shown by an expanding area of redness on the skin. This blood sample was withdrawn from the vein by a physician and then forwarded to a microbial lab for the usual serological tests. The evidence of antibodies by IgG tests indicated a recent infection by Borrelia burgdorferi sensu lato ( $B$. burgdorferi s. s, B. garnii, B. afzelii, B. spielmanii). This result agreed well with that of the gas chromatographic analysis as shown by the emitted hydrogen peak in the chromatogram in Figure 2b. Electron micrographs performed with the cultured specimen prepared $48 \mathrm{~h}$ later, are shown in Figure 2-a,b using trans electron microscopy (TEM). We here identified the successful enrichment of spirochetes resembling Borrelia l-forms after 7 days of cultivation and $48 \mathrm{~h}$ of shipment (Figure 1-Ba, sample1).

As soon as bacterial infection by Borrelia species are detected by HS-GC-analysis of blood a suitable antibiotic therapy should commence. In accordance to the current guidelines tetracyclines are recommended as first-line treatment. However, opinions on the necessary dosage and the adequate length of therapy varies widely between many experts. This is due to the missing control about the effect of such a therapy. Borrelia bacteria in blood are in general identified indirectly by serological tests by determining antibodies. However even after completion of the infection the antibodies persist in blood. Moreover, these serological tests are notoriously unreliable. The following example represents such a typical case. Some weeks after having been bitten by the tick, the patient complained about typical symptoms of a tick-borne infection including myalgia. A blood sample was then analyzed by the GCmethod and the hydrogen peak indicated a possible infection by Borreliella (Figure 3-Aa).

The result from a simultaneously performed serological test, however, was unclear because antibodies by ELISA test were not found, which may be due to a short persistence of the clinical symptoms. But due to the hydrogen peak in the resulting chromatogram from HS-GC-analysis, a therapy with doxycycline started with a daily dosage of $200 \mathrm{mg}$ doxycycline. After nine days of treatment, a hydrogen peak in the chromatogram still implicated the therapy as insufficient. Seven days later after administration of a total doxycycline dosage of $3200 \mathrm{mg}$, the hydrogen peak was eliminated (Figure 3-Ab). An additional control analysis performed a few days later, implicated the successful end of the therapy as the missing hydrogen peak of the blood sample was identical to the blank of a sterilized cotton ball in the nutrient broth. Since the emission of hydrogen is a direct proof for infected blood, the progress of a therapy can be monitored, and the successful therapeutic outcome might thus be determined.

\section{Borreliosis therapy with the natural antibiotic in garlic tablets.}

The first line treatment of borreliosis is based on antibiotics such as doxycycline used for adults and amoxicillin or cefuroxime acetyl for adults and children. These antibiotics work sufficiently well when administrated at the early stages of borrelia infection but not at its late stages [13-18] However, some patients experience adverse effects with chemical antibiotics which may range from undesired to life threatening. Furthermore, in course of the treatment regimen resistances may rise. Some natural products, particularly essential oils, are known for their antibiotic effect $[7,19]$ and cardea, cress seed, propolis and several more have been examined in this work (data not shown). From these, two natural products have emerged as most effective: oil of cloves and garlic. The application of oil of cloves, however, poses difficulties unless the active agent is isolated and identified. Garlic on the other hand is available as various preparations for ingestion, such as pills, tablets, dragees or extracts. In this work tablets with pulverized garlic were examined for their suitability in replacing rough garlic.

The antibiotic property of garlic had already been described by Louis Pasteur and was investigated for its suitability against Borrelia in vitro [20]. Particularly allicin and related sulphur compounds are known for their anti-microbial activity [21]. Garlic therefore may be considered a natural antibiotic that furthermore has also been demonstrated as effective against some antimicrobial-resistant bacteria. For these reasons, garlic was selected in this work to investigate whether it can be applied against Lyme borreliosis in vivo. Figure 3-B displays the results of the therapy applied to an infected patient with garlic tablets. After a 7day therapy regimen with a daily dosage of $900 \mathrm{mg}$ the hydrogen peak has disappeared and apparently the Borreliella bacteria in blood have been eliminated. The therapy was continued with a daily dosage of $300 \mathrm{mg}$ and even 30 days later was the result of GC-analysis the same as given by chromatogram 2 in Figure 3-B and thus identical with that of a healthy person.

These results demonstrate that garlic, even applied as tablets, can be an effective drug against borreliosis, comparable to the standard antibiotic treatment. However, it should be mentioned that the short therapy span in the above-mentioned example doesn't apply to every case. We have found for other patients longer therapeutic regimens between one to three weeks to be necessary. The difference might be caused by a different bacterial load in blood or inter-individual differences such as immune status or pre-existing conditions. Each therapy should therefore be controlled regularly until its successful end is confirmed. The method described allows complete control of a Borreliella-infection starting with the proof a contaminated tick up to the successful end of a suitable therapy.

The actual absence of Borreliella in blood does not necessarily indicate complete bacteria eradication because they may change into inactive round bodies (stationary phase, cysts, L-forms (Figure 2) and enter other parts of the human body such as skin or cartilage, where up to now they cannot be detected neither by the described GC-method nor with the standard serological tests, 
but may be treated by the garlic therapy [18]. From these hidden places the bacteria may change back to the motile helical form and can migrate into blood where they can be recognized and combated again. Such regularly repeated attacks are well known and described as persistent Lyme disease and are often enhanced if the immune system is weakened by a new infection or by a new disease as shown by the following example. The patient suffering from hidden persistent Lyme disease experienced an infection by Noro virus. Figure $3 \mathrm{Ca}$ is the result of blood analysis before this viral infection, apparently with no bacteria in blood. One week after Noro virus infection the hydrogen peak (peak 1, Figure. 3 - $\mathrm{Cb}$ ) from blood analysis indicated the repeated emergence of Borreliella, apparently due to the weakened immune system. A 12day garlic therapy with a daily dosage of $1800 \mathrm{mg}$ pulverized garlic the bacterial load in the blood (peak 2, Figure 3-Cb) was still not enough to eliminate all bacteria. Only after an extended additional 9-day therapy with $900 \mathrm{mg}$ /day the bacteria were eradicated in blood and the GC-test was identical with that shown in Figure 3-Ca, as was a control test two weeks later.
A

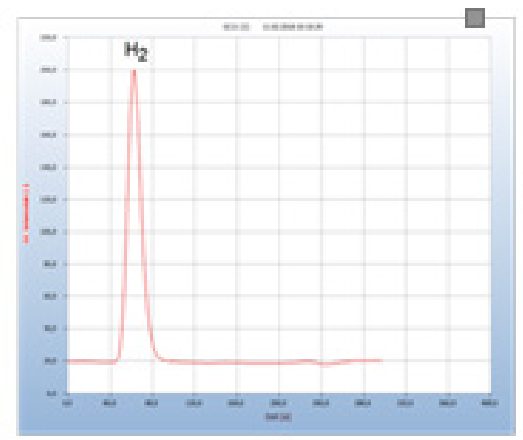

(a)

B

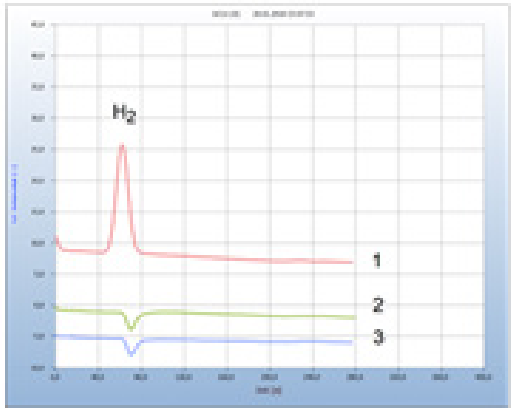

C

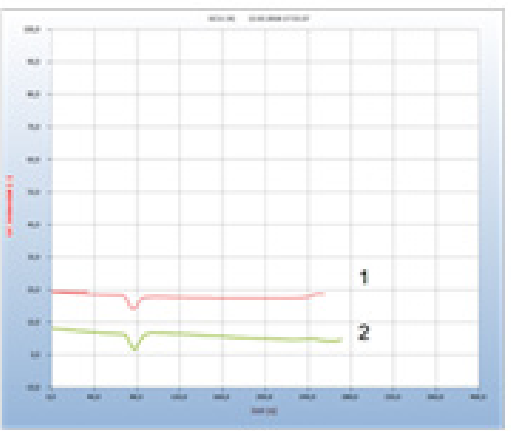

(a)

Q.

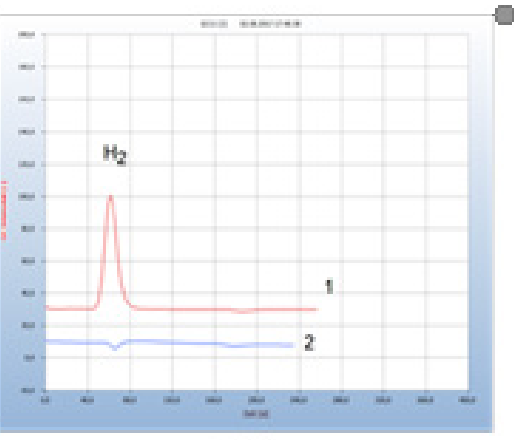

(b)

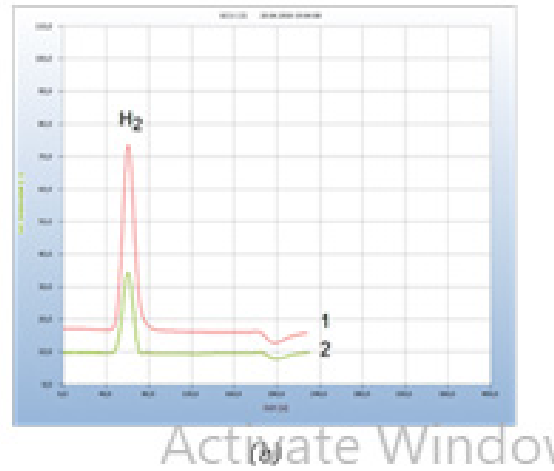

Figure 3: GC analysis of blood samples under different conditions A) GC analysis of blood samples pre and post doxycycline treatment. (a) Analysis of blood after tick infection. (b) Analysis of blood after doxycycline therapy with 200 mg of doxycycline daily; 1 : after 9 days; 2 : after 16 days. B) GC analysis of blood samples pre and post therapy with garlic tablets. Efficacy of therapy with garlic tablets. 1: infected blood before therapy; 2: blood after 7 days therapy; 3: blank from cotton ball in medium. C) GC analysis of blood samples pre and post Noro virus infection. (a) GC analysis of blood nutrient before infection with Noro virus; 1 : blood before Noro virus infection; 2 : blank from cotton ball in nutrient medium. (b) Influence of Noro virus infection; 1: blood 8 days from beginning of Noro-disease, 2: blood 12 days later after application of garlic tablets.

\section{Discussion}

Static HS-GC successfully detects hydrogen emitted from bacterial cultures. We here applied a small, and low-cost device, GCAK-11 to detect the bacterial contamination in ticks and in patients derived blood samples, respectively. Moreover, this device has been successfully applied to test and evaluate the effect and treatment by standard antibiotics as well as phytochemicals such as clove oil and ingredients from garlic extracts. As a conclusion, this GC-method appears to be valid for both: diagnosis and therapy to control 
facultative anaerobes spirochetes including Borreliella. In contrast to acute Borreliella infection, chronic borreliosis has been found to be resistant to even extended antibiotic treatment, but promising effects have already been attributed to treatment regimen using phytochemicals. Using in vitro culture of Borrelia, the group headed by Eva Sapi summarized effects on different forms of pathogenic Borrelia [22], (another comprehensive report has been published by Goc and colleagues [23].

The here described GC-method can be used to test more natural substances as well as combinations for their biocide properties against facultative anaerobes, e.g. Borreliella. Knowledge of the actual bacterium or the composition of such a bacteria mix in case of Lyme disease is of less interest, if an effective therapy would eradicate all of them. The patient is interested only to get the most effective therapy and to know how long it takes. It is the advantage of our method that all those hydrogen emitting pathogens, such as Borreliella are comprised in our test and that it is not confined to $B$. burgdorferi. The HS-GC technique has the unique advantage that the whole procedure is carried out in hermetically closed vials, already beginning with sample preparation. When the samples are inserted into the nutrient medium the headspace vials are capped with PTFE-lined septa and sealed with a crimped closure and any contamination by invasion of external microbes is thus excluded. The septum is then pierced with the needle of a gas tight syringe and an aliquot of the headspace gas is withdrawn and injected into the gas chromatograph.

The septum remains tight and several gas samples can be analyzed at given time intervals thus enabling kinetic measurements to be carried out. After analysis, the closed vials with their contents can be sterilized at the necessary high temperature and then safely discarded. Thus, the personnel in a laboratory never meet pathogens. The described method can be carried out with all commercially available gas chromatographs, even including simple low-cost instruments up to expensive and fully automated instruments. The results presented here were obtained with a very cheap gas chromatograph for students equipped with a standard packed column, a thermal conductivity detector and can be operated at room temperature with ambient air as carrier gas fed onto the column by an aquarium pump. With commercially available instruments on the other hand, up to 100 samples can be processed automatically. This automated technique is thus particularly suited for screening purposes to analyze a large number of samples, for example to search for alternative antibiotics in the event of antibiotic resistance, and even for adaptation to an individual patient. The electrical signal generated by an automated headspace instrument lends itself to automated computer evaluation and documentation.

A systematic diagnosis and therapy may proceed according to the following scheme:
1. A tick after having been removed from the body is analyzed immediately as to whether it is contaminated by Borreliella or by other pathogens.

2. If the tick has been found contaminated, analysis of the blood shows whether the infection was transmitted to the blood, since not in all cases are the bacteria transferred.

3. If bacteria are found in the blood, a suitable therapy should be started immediately.

4. The progress of the applied therapy is permanently controlled until it is successfully finished.

5. Even after the therapy is finalized, it is recommended to repeat this test regularly, particularly in the case of persistent borreliosis or newly emerging health complaints.

In case this test confirms borreliosis the physician will in any case initiate the common and well accepted antibiotic treatment such as doxycycline. In case of serious side effects, alternative natural antibiotic may help. Herbs are commonly safe, while chemical antibiotics may damage the microbiome. Particularly chronic use of antibiotics may disrupt the immune system and the protective biofilm in the colon. The possibility of using a natural product as an alternative antibiotic was examined on the example of garlic in this study, but other natural antibiotics may work as well $[8,19]$. The garlic tablet preparations were found to be advantageous since no prescription is required and bacterial testing could be performed independently of a caring physician. Further biotechnological developments of the HS-GC-method may be addressed in the future.

\section{Funding}

This work was supported by the Baden-Württemberg Stiftung

\section{Author Contributions}

Conceptualization, B.K; methodology, B.K. and M.S.; investigation L.R. and L.B: validation T.H.; writing-original draft preparation B.K.; writing-review and editing M.S. and T.H.

\section{Acknowledgment}

We thank Paul Walther, head of the Central Facility for Electron Microscopy, Ulm University, for providing support in electron microscopy and image processing.

\section{Conflicts of Interest}

The authors declare no conflict of interest. The funders had no role in the design of the study; in the collection, analyses, or interpretation of data; in the writing of the manuscript, or in the decision to publish the results.

\section{Ethical statement}

All subjects gave their consent before they participated in this study. 


\section{References}

1. Bassette R, Claydon TJ (1965) Characterization of some bacteria by gas chromatographic analysis of head space vapours from milk cultures. J Dairy Sci (48): 775-779.

2. Seifert S, Böhnel H, Gierke S, Heine A, Hoffman D, et al. (1986) Identification of anaerobic bacteria by fatty acid pattern recognition. International Laboratory pp: 46-56.

3. Heitefuß S, Heine A, Seifert S (1990) Identification of anaerobic bacteria by determination of non-volatile dicarboxylic acids after derivatization. J Chromatogr Biomed Appl (532): 374-379.

4. Bean HD, Dimandja JM, Hill JE (2012) Bacterial volatile discovery using solid phase microextraction and comprehensive two-dimensional gas chromatography-time-of-flight mass spectrometry. J Chromatogr B, Analytical technologies in the biomedical and life sci (901): 41-46.

5. Kolb B, Ettre LS (2006) Static Headspace-Gas Chromatography: Theory and Practice, $2^{\text {nd }}$ edn. Wiley-Inter science, pp. 286-289.

6. Goc A, Rath M (2016) The anti-borrelia efficacy of phytochemicals and micronutrients: an update. Ther adv infect dis 3(3.4): 75-82.

7. Kolb BK, Riesterer L, Bier L, Widenhorn AM (2019) Proof of bacteria and the activity of chemical and natural antibiotics by headspace gas chromatography. Journal of Analytical Science and Technology 10:1-9.

8. KolbB,WidenhornAM, RiestererL,BierL(2017)Gaschromatographischer Test zum Nachweis von Bakterien und zur Wirksamkeit von Antibiotic am Fallbeispiel von Borreliose, Borreliose Wissen 36: 3-6.

9. Donta ST (2012) Issues in the diagnosis and treatment of Lyme disease. The Open Neurol J 6: 140-145.

10. Marques AR (2015) Laboratory diagnosis of Lyme disease: advances and challenges. Infect Dis Clin North Am 29(2): 295-307.

11. Wormser GP, McKenna D, Scavarda C, Cooper D, El Khoury MY, et al (2019) Co-infections in Persons with Early Lyme Disease. Emerging Infectious Diseases 25(4): 748-752.
12. Cutler SJ, Ruzic-Sabljic E, Potkonjak (2017) Emerging borreliaeExpanding beyond Lyme borreliosis. Mol Cellular Prob (31): 22-27.

13. Embers ME, Barthold SW, Borda JT, Bowers L, Doyle L, et al. (2012) Persistence of Borrelia burgdorferi in Rhesus Macaques following Antibiotic Treatment of Disseminated Infection. PLOS ONE 7(1): 1-12.

14. Eppes SC, Childs JA (2002) Comparative study of cefuroxime axetil versus amoxicillin in children with early Lyme disease. Pediatrics 109(6): 1173-1177.

15. Donta ST (2002) Late and chronic Lyme disease. Med Clin North Am 86(2): 341-349.

16. Donta ST (2007) Lyme disease guidelines it's time to move forward. Clin infect dis: an official publication of the Infectious Diseases Society of America 44(8):1134-1135.

17. Massarotti EM, Luger SW, Rahn DW, Messner RP, Wong JB, et al. (1992) Treatment of early Lyme disease. Am J Med 92(4): 396-403.

18. Stanek G, Wormser GP, Gray J, Sterle F (2012) Lyme borreliosis. Lancet (London, England) 379(9814): 461-73.

19. Feng J, Zhang S, Shi W, Zubcevik N, Miklossy J, et al. (2017) Selective Essential Oils from Spice or Culinary Herbs Have High Activity against Stationary Phase and Biofilm Borrelia burgdorferi. Front Med 4:169.

20. Feng J, Shi W, Miklossy J, Tauxe GM, McMeniman CJ, et al. (2018) Identification of Essential Oils with Strong Activity against Stationary Phase Borrelia burgdorferi. Antibiotics (Basel, Switzerland) 7(4): 89.

21. Ankri S, Mirelman D (1999) Antimicrobial properties of allicin from garlic. Microbes Infect. 1(2): 125-129.

22. Sapi E, Kaur N, Anyanwu S, Luecke DF, Datar A, et al. (2011) Evaluation of in-vitro antibiotic susceptibility of different morphological forms of Borrelia burgdorferi. Infect Drug Resist 4: 97-113.

23. Goc A, Niedzwiecki A, Rath M (2015) In vitro evaluation of antibacterial activity of phytochemicals and micronutrients against Borrelia burgdorferi and Borrelia garinii. Journal of Applied Microbiology 119:1561-1572. 